\title{
Domain Wall Spin Structures in 3d Metal Ferromagnetic Nanostructures
}

\author{
M. Laufenberg ${ }^{1}$, M. Kläui ${ }^{1}$, D. Backes ${ }^{1,2}$, W. Bührer ${ }^{1}$, H. Ehrke ${ }^{1,5}$, \\ D. Bedau ${ }^{1}$, U. Rüdiger ${ }^{1}$, F. Nolting ${ }^{2}$, L. J. Heyderman ${ }^{2}$, S. Cherifi ${ }^{3}$, \\ A. Locatelli ${ }^{3}$, R. Belkhou ${ }^{3}$, S. Heun ${ }^{3}$, C. A. F. Vaz ${ }^{4}$, J. A. C. Bland ${ }^{4}$, \\ T. Kasama ${ }^{5}$, R. E. Dunin-Borkowski ${ }^{5}$, A. Pavlovska ${ }^{6}$, and E. Bauer ${ }^{6}$ \\ 1 Fachbereich Physik, Universität Konstanz, 78457 Konstanz, Germany \\ 2 Paul Scherrer Institut, 5232 Villigen PSI, Switzerland \\ 3 Nanospectroscopy Beamline, Sincrotrone Trieste, 34012 Basovizza/Trieste, \\ Italy \\ ${ }^{4}$ Cavendish Laboratory, University of Cambridge, Cambridge CB3 0HE, U.K. \\ 5 Department of Materials Science and Metallurgy, University of Cambridge, \\ Cambridge CB2 3QZ, U.K. \\ 6 Department of Physics and Astronomy, Arizona State University, Tempe, \\ Arizona 85287-1504, USA
}

\begin{abstract}
In this article, a comprehensive study of head-to-head domain wall spin structures in $\mathrm{Ni}_{80} \mathrm{Fe}_{20}$ and Co nanostructures is presented. Quantitative domain wall type phase diagrams for $\mathrm{NiFe}$ and $\mathrm{Co}$ are obtained and compared with available theoretical predictions and micromagnetic simulations. Differences to the experiment are explained taking into account thermal excitations. Thermally induced domain wall type transformations are observed from which a vortex core nucleation barrier height is obtained. The stray field of a domain wall is mapped directly with sub-10 $\mathrm{nm}$ resolution using off-axis electron holography, and the field intensity is found to decrease as $1 / r$ with distance. The magnetic dipolar coupling of domain walls in $\mathrm{NiFe}$ and Co elements is studied using X-ray magnetic circular dicroism photoemission electron microscopy. We observe that the spin structures of interacting domain walls change from vortex to transverse walls, when the distance between the walls is reduced. Using the measured stray field values, the energy barrier height distribution for the nucleation of a vortex core is obtained.
\end{abstract}

\section{Introduction}

Domain walls in nanoscale ferromagnetic elements are in the focus of interest because of their potential for applications in a variety of fields such as magnetic logic [1] as well as data storage $[2,3]$ and due to their associated fundamental physical effects [4-10]. Magnetoresistance effects related with domain walls have been investigated in recent years $[4-6,11,12]$. Domain wall motion induced by external fields [7-9] has been studied and wall mobilities and depinning fields [10] have been determined. Current induced domain wall motion and the underlying spin torque effect have been the subject of rising interest recently $[13-15]$ with investigation of critical current densities and of domain wall velocities. The resistivity of a domain wall, its mobility, the 
depinning fields and critical currents as well as the spin torque effect depend critically on the wall spin structure $[1,4-10,13-15]$. In this field of research, temperature and heating effects play a key role [16-19], in particular the wall spin structure was predicted to depend on the temperature [19], which calls for studies at variable temperatures. Ring elements have proven to be a useful geometry for the investigation of domain walls since they can easily be created and positioned by applying an external uniform magnetic field. Ferromagnetic rings can be in the flux closure vortex state or in the onion state characterized by $180^{\circ}$ head-to-head and tail-to-tail domain walls [20], which can be of either vortex or transverse type [21] with the spin structures shown in Figs. 1(a) and (b), respectively.

A strong influence of domain wall interaction on the switching of magnetic elements was found recently, when interaction-induced collective switching of adjacent elements was observed for small spacings [22,23]. Such switching is dominated by domain wall motion and can only be understood with a detailed knowledge of the interacting domain walls' spin structures. Theoretically, the energies of the two wall types are different when interacting with an extemal field. We therefore expect the dipolar coupling to affect the two wall types in different ways and coupling-induced transitions from one domain wall type to another may occur [21]. A deeper understanding of the energetics involved is only possible if the stray fields of domain walls are determined quantitatively.

In this paper, we review our recent work on domain wall spin structures in mesoscopic NiFe and Co ring elements. For investigation of the spin structure of domain walls, we use high-resolution imaging by X-ray magnetic circular dichroism photoemission electron microscopy (XMCD-PEEM) as well as offaxis electron holography providing sub- $10 \mathrm{~nm}$ resolution. In particular, we systematically study the spin structure of head-to-head domain walls at variable temperature. We present the phase diagrams and compare the findings with available theoretical calculations and the results of micromagnetic simulations. We derive a qualitative temperature dependence of the phase boundary and extract the energy barrier height for the vortex nucleation. Having comprehensively explored the spin structures of isolated domain walls, we study the interaction of adjacent walls and their dipolar coupling. By correlating the domain wall spin structure changes due to increasing edge-to-edge spacing between neighboring elements with a quantitative measurement of the domain wall stray field, we obtain a direct measure of the energy barrier distribution for the vortex core nucleation in our samples.

Arrays of $5 \times 5$ polycrystalline Co and NiFe rings with different thicknesses and widths were fabricated as described in [24,25]. For the first set of samples, the edge-to-edge spacing between adjacent rings was more than twice the diameter to prevent dipolar interactions which might otherwise influence the domain wall type. To determine the spin structure of the domain walls as a function of the ring geometry, the samples were imaged using XMCDPEEM [26]. Arrays of $350 \mathrm{~nm}$ wide rings with edge-to-edge spacings down to $10 \mathrm{~nm}$ were fabricated to investigate different dipolar coupling strengths 


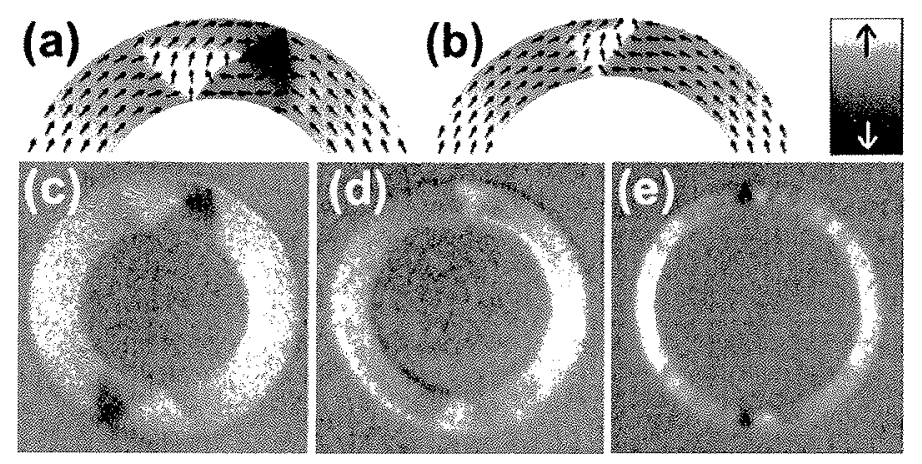

Fig. 1. (from [30]) Spin structure of (a) a vortex and (b) a transverse wall simulated using OOMMF. PEEM images of (c) $30 \mathrm{~nm}$ thick and $530 \mathrm{~nm}$ wide $(D=2.7 \mu \mathrm{m})$, (d) $10 \mathrm{~nm}$ thick and $260 \mathrm{~nm}$ wide $(D=1.64 \mu \mathrm{m})$, and (e) $3 \mathrm{~nm}$ thick and $730 \mathrm{~nm}$ wide $(D=10 \mu \mathrm{m}) \mathrm{NiFe}$ rings. The gray scale indicates the direction of magnetic contrast

between domain walls in adjacent rings and resulting domain wall types. For the transmission off-axis electron holography experiments, 3/4-rings were patterned from $27 \mathrm{~nm}$ thick Co films on $50 \mathrm{~nm}$ thick SiN membranes as detailed in [27]. Open rings rather than full rings were grown on the fragile membranes in order to facilitate the lift-off process which cannot be assisted by ultrasound. In order to obtain quantitative information about stray fields, Co samples were investigated by off-axis electron holography $[28,29]$. Co was chosen rather than NiFe for this investigation due to its higher saturation magnetization and therefore higher stray field.

\section{Domain Wall Spin Structure}

\subsection{Domain Wall Phase Diagrams}

In Fig. 1, we present PEEM images of (c) a thick and wide NiFe ring, (d) a thin and narrow ring, and (e) an ultrathin ring measured at room temperature. The domain wall type was systematically determined from PEEM images for more than 50 combinations of ring thickness and width for both NiFe and Co and the quantitative phase diagrams shown in Figs. 2(a) and (c) were extracted. The phase diagrams exhibit two phase boundaries indicated by solid lines between vortex walls (thick and wide rings, squares), transverse walls (thin and narrow rings, circles), and again vortex walls for ultrathin rings.

Now we first discuss the upper boundary shown in Figs. 2(a,c). Theoretically this phase boundary was investigated by McMichael and Donahue [21]. They calculated the energies for a vortex and a transverse wall and determined the phase boundary by equating these two energies. The calculated 

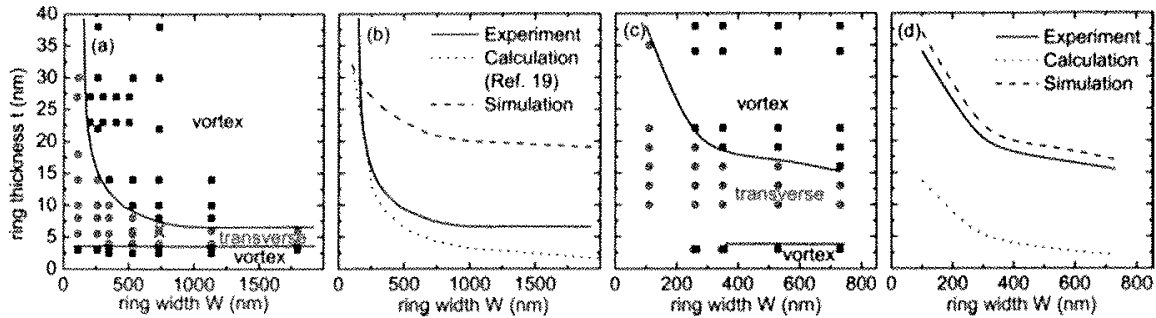

Fig. 2. (Color online, partly from $[30,31]$ ) Experimental phase diagrams for headto-head domain walls in (a) $\mathrm{NiFe}$ and (c) Co rings at room temperature. Black squares indicate vortex walls and circles transverse walls. The phase boundaries are shown as solid lines (online color). (b, d) Comparison of the upper experimental phase boundary (solid lines) with results from calculations (dotted lines) and micromagnetic simulations (dashed lines). The thermally activated wall transitions shown were observed for the ring geometry marked with a red cross (online color) in (a) $(W=730 \mathrm{~nm}, t=7 \mathrm{~nm})$

phase boundary (dotted lines in Figs. 2(b,d)) is of the form $t \cdot W=C \cdot \delta^{2}$ where $\delta$ is the exchange length and $C$ a universal constant. It is shifted to lower thickness and smaller width compared to the experimental boundary (solid lines in Figs. 2(b,d)). This discrepancy can be understood by taking into account the following: The calculations [21] compare total energies and therefore determine the wall type with the absolute minimum energy as being favorable. In the experiment, the wall type was investigated after saturation of the ring in a magnetic field and relaxing the field to zero. During relaxation, first a transverse wall is formed reversibly [32]. For the formation of a vortex wall, an energy barrier has to be overcome to nucleate the vortex core. So the observed spin structure does not necessarily constitute the absolute minimum energy, but transverse walls can be observed for combinations of thickness and width where they constitute local energy minima even if a vortex wall has a lower energy for this geometry. To shed further light onto this, we have simulated the experiment by calculating the domain wall spin structure after reducing an externally applied field stepwise using the OOMMF code [33] (for NiFe: $M_{\mathrm{s}}=800 \times 10^{3} \mathrm{~A} / \mathrm{m}, A=1.3 \times 10^{-11} \mathrm{~J} / \mathrm{m}$; for $\mathrm{Co}: M_{\mathrm{s}}=1424 \times 10^{3} \mathrm{~A} / \mathrm{m}, A=3.3 \times 10^{-11} \mathrm{~J} / \mathrm{m}$; for both: damping constant $\alpha=0.01$, cell size $2-5 \mathrm{~nm}$ ). The simulated boundary is shifted to higher thickness and larger width compared to the experiment. This we attribute to the fact that thermal excitations help to overcome the energy barrier between transverse and vortex walls in case of the room temperature experiment, while they are not taken into account in the $0 \mathrm{~K}$ simulation. So we can expect that for temperatures above room temperature the upper phase boundary is shifted to lower thickness, in other words, that transverse walls formed at room temperature change to vortex walls with rising temperature. This means that with rising temperature the experimental phase boundary 

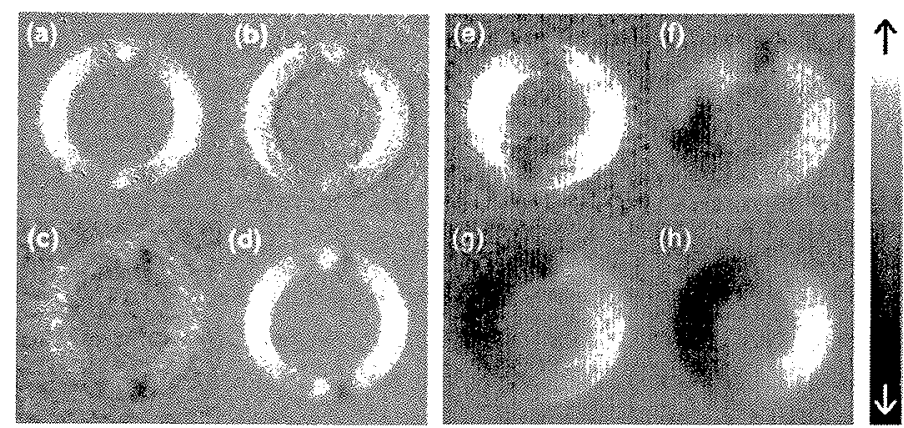

Fig. 3. (partly from [30]) PEEM images of a $7 \mathrm{~nm}$ thick and $730 \mathrm{~nm}$ wide ring imaged during a heating cycle at temperatures of $(\mathbf{a}, \mathrm{d}) T=20^{\circ} \mathrm{C}$ (before and after heating, respectively), (b) $T=260^{\circ} \mathrm{C}$, and (c) $\mathcal{T}=310^{\circ} \mathrm{C}$ (estimated errors are $\pm 10 \mathrm{~K}$ ). Due to heating, rings (here (e): $7 \mathrm{~nm}$ thick, $1135 \mathrm{~nm}$ wide with two vortex walls) can attain either (g) a vortex state with a $360^{\circ}$ domain wall or (h) the vortex state. The intermediate state, where one wall is displaced, is shown in (f). The gray scale indicates the direction of magnetic contrast

approaches the theoretical one since the walls attain the energetically lower spin structure.

\subsection{Thermally Activated Domain Wall Transformations}

In order to corroborate this explanation for the difference between the experiment on the one hand and calculations and simulations on the other hand, we have performed temperature dependent XMCD-PEEM studies. Figure 3 shows an image series of a $7 \mathrm{~nm}$ thick and $730 \mathrm{~nm}$ wide $\mathrm{NiFe}$ ring (geometry marked by a cross in Fig. 2(a)) for different temperatures of (a, d) $T=20^{\circ} \mathrm{C}$ (before and after heating), (b) $T=260^{\circ} \mathrm{C}$, and (c) $T=310^{\circ} \mathrm{C}$. Transverse walls are formed (a) during saturation in a magnetic field and relaxation before imaging. At first, heating does not influence the spin structure of the domain walls as shown in (b), only the image contrast becomes weaker because imaging is more difficult at higher temperatures due to drift problems and decreasing magnetization. At a transition temperature between $T=260^{\circ} \mathrm{C}$ and $T=310^{\circ} \mathrm{C}$ corresponding to a thermal energy between $6.7 \times 10^{-21} \mathrm{~J}$ and $8.0 \times 10^{-21} \mathrm{~J}$, the transverse walls change to vortex walls (c), so that a domain wall spin structure was created which is not accessible for the same ring geometry by only applying uniform magnetic fields. Figure 3(d) confirms that the vortex wall is stable during cooling down. This means that both domain wall types are (meta-)stable spin configurations and therefore constitute local energy minima at room temperature for this geometry. These PEEM experiments directly show that the position of the upper experimental phase boundary is temperature dependent and is shifted to lower thickness and width with increasing temperature. These results thus confirm the hypothesis about the discrepancy between experiment and theory put forward 
before: Both domain wall types constitute local energy minima, with the transverse wall attained due to the magnetization process, even if a vortex wall has a lower energy. Experimentally, we directly observe thermally activated crossing of the energy barrier between high energy transverse and low energy vortex walls.

It should be mentioned however, that the flux closure vortex state of the ring without any domain walls and with the magnetization aligned everywhere along the ring perimeter is the energetically most favorable state. Many rings attain this state when the temperature is increased as shown in Figs. 3(e-h). In order to observe the wall type transformations shown in Figs. 3(a-d), it is therefore necessary, that the energy barrier between transverse and vortex walls is lower than the barrier for the transition to the vortex state of the ring. This critically depends on imperfections of the ring microstructure which can serve as pinning centers and stabilize a domain wall.

It can be seen by comparing the boundaries for NiFe and Co in Fig. 2, that for NiFe the calculations [21] fit the experiment better than the simulations while for Co the opposite is true. The energy barrier between a transverse and a vortex wall can be overcome more easily in the case of NiFe rather than $\mathrm{Co}$, so that transverse walls created are more likely to be retained at a certain temperature in a Co ring than in a NiFe ring with analogous dimensions. This is consistent with the observation that in NiFe there is a more abrupt change between transverse and vortex walls with varying geometry than in Co.

\subsection{Walls in Thin and Wide Structures - Limits of the Description}

We turn now to the discussion of the low thickness regime of the phase diagrams shown in Fig. 2, where a second phase boundary between 3 and $4 \mathrm{~nm}$ is found both for NiFe and for Co. In terms of energetics, this is not expected because the calculations [21] show that a transverse wall has a lower energy than a vortex wall in this thickness regime. But these calculations assume a perfect microstructure and do not take into account morphological defects such as the surface roughness. Holes, which might serve as nucleation centers for the vortex wall formation, were not observed in atomic force microscopy images. However, this does not exclude a spatial modulation of magnetic properties [37] such as the exchange or the saturation magnetization, which could locally allow for a stronger twisting of adjacent spins. Thus a vortex wall would be energetically more favorable in this thickness regime only due to imperfections of the microstructure or the morphology.

In the thin samples investigated, a ripple domain formation [38] is observed as shown in Figs. 4(a,b) (see also Fig. 1(e)). This can be attributed to statistical variations of the anisotropy of individual grains. Consequently, this phenomenon is more pronounced in the polycrystalline Co structures, in 


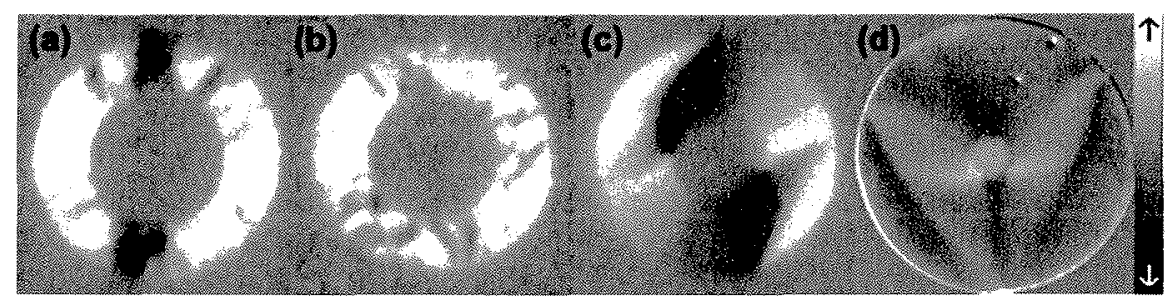

Fig. 4. Limiting cases of the structures investigated: $3 \mathrm{~nm}$ thick and $1.8 \mu \mathrm{m}$ wide (a) $\mathrm{NiFe}$ and (b) Co rings showing ripple domain formation, (c) $6 \mathrm{~nm}$ thick and $3 \mu \mathrm{m}$ wide ring with distorted transverse walls, and (d) $10 \mathrm{~nm}$ thick and $2.1 \mu \mathrm{m}$ wide disc-like NiFe ring with $700 \mathrm{~nm}$ inner diameter in the triangle state (for detailed explanations of the contrast see [34-36]). The gray scale indicates the direction of magnetic contrast

which individual grains exhibit a non-negligible anisotropy compared to the weak anisotropy in $\mathrm{NiFe}$.

The description in the frame of these phase diagrams is however limited by the width and thickness of the structure. In rings wider than $\approx 1.5 \mu \mathrm{m}$, we observe more complicated domain wall spin structures like distorted transverse walls (Fig. 4(c)). Wide rings with a hole in the center exhibit a disc-like behavior with a triangle state as shown in Fig. 4(d). This type of structure is discussed in more detail in [34-36]. In very thick elements double vortex walls were reported recently [39], which can also constitute (meta-)stable configurations in the thickness regime investigated here, as it turned out from experiments on current-induced domain wall transformations [40]. Recent theoretical calculations [41] propose to distinguish between symmetric and asymmetric transverse walls which is not done here, because both types are difficult to distinguish experimentally and sample irregularities can influence the detailed wall spin structure.

\section{Domain Wall Coupling Energetics}

\subsection{Coupling Between Adjacent Domain Walls}

After saturating with a magnetic field and relaxing the field, rings attain the onion state characterized by two head-to-head domain walls as shown before (see also Fig. 5(a)). An array of 25 rings in the onion state exhibits 50 walls in total. The domain walls inside the array interact with adjacent walls via their stray fields. Only 10 walls, which are located at the two opposite edges (top and bottom edges in Figs. 5(b) and (c)) of the array and therefore have no neighboring rings, are not influenced by stray fields of an adjacent wall. For all experiments, ring thickness and width were chosen such that isolated 


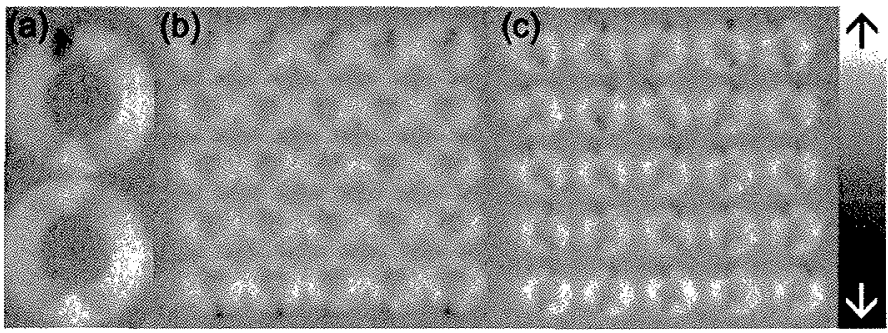

Fig. 5. (from [42]) (a) A high-resolution XMCD-PEEM image of two rings in the onion state, after saturation with an external field in the vertical direction and relaxation. White and black contrasts correspond to the magnetization pointing up and down, respectively. A non-interacting vortex wall (top) and three interacting transverse walls are visible. Overview images of an array of $27 \mathrm{~nm}$ thick and $350 \mathrm{~nm}$ wide NiFe rings with an edge-to-edge spacing of (b) $40 \mathrm{~nm}$ and (c) $500 \mathrm{~nm}$, respectively. The transition from $100 \%$ transverse walls inside the array for narrow spacings (b) to close to $0 \%$ for large spacings (c) can be clearly seen. Since domain walls at the top and bottom edges of the array do not interact with adjacent walls, they are vortex walls for all spacings investigated

rings of this geometry exhibit vortex walls according to the phase diagram presented above.

Figure 5 shows XMCD-PEEM images of arrays of $27 \mathrm{~nm}$ thick NiFe rings with (b) $40 \mathrm{~nm}$ and (c) $500 \mathrm{~nm}$ edge-to-edge spacing, respectively, as well as a high-resolution image (a) presenting both wall types. Vortex walls can be easily identified by black and white contrast which occurs because all magnetization directions corresponding to the full grey scale are present in a vortex (Fig. 5(a), top). In contrast, transverse walls exhibit the characteristic grey-white-grey contrast with the triangular spin structure in their center (Fig. 5(a), bottom).

In Fig. 6(a), we show the percentage of transverse walls inside the array as function of the edge-to-edge spacing for $27 \mathrm{~nm}$ thick $\mathrm{NiFe}$ rings (black squares) extracted from images of the type shown in Fig. 5. A decreasing number of transverse walls is found with increasing spacing. Domain walls at the edges of the arrays are vortex walls irrespective of the spacing due to the absence of dipolar coupling with adjacent walls. The data points for infinite spacings in Fig. 6(a) result from these domain walls. The transverse to vortex transition is characterized by a (10-90\%)-width of the switching distribution of $w=(65 \pm 9) \mathrm{nm}$ and a center at $r_{\mathrm{c}}=(77 \pm 5) \mathrm{nm}$. In Fig. 6(a), red triangles show a similar transition for $30 \mathrm{~nm}$ thick Co rings with $w=(328 \pm 130) \mathrm{nm}$ and $r_{\mathrm{c}}=(224 \pm 65) \mathrm{nm}$.

In order to explain these results, we first consider the process of domain wall formation in an isolated ring. When relaxing the applied external field from saturation, transverse walls are initially formed. In order to create a vortex wall, a vortex core has to be nucleated. This hysteretic transition from 

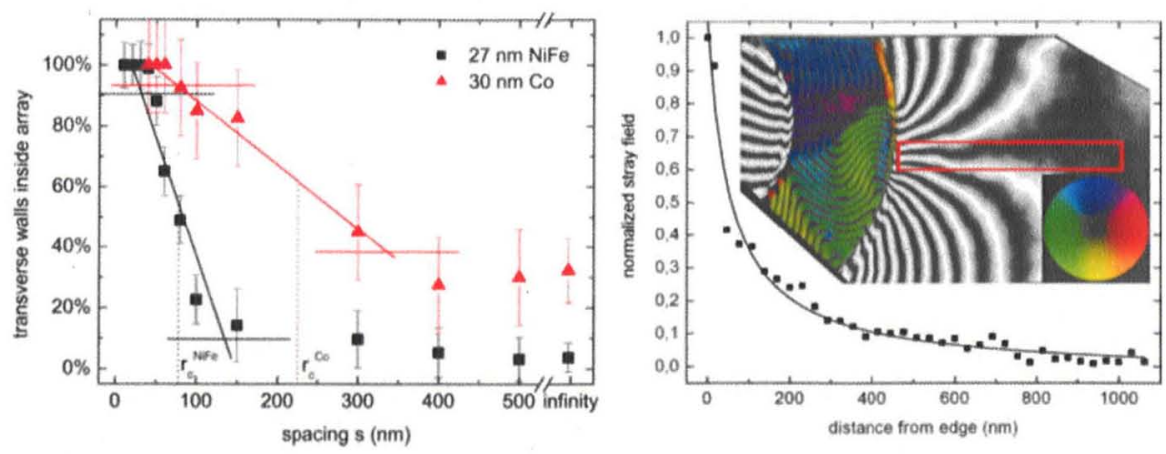

Fig. 6. (Color online, from [42]) (a) Percentage of transverse walls inside a ring array as function of edge-to-edge spacing. Black squares are for $27 \mathrm{~nm} \mathrm{NiFe}$, and red triangles (online color) for $30 \mathrm{~nm}$ Co, respectively. The error bars represent the absolute statistical error $1 / \sqrt{n}$ due to the finite number $n$ of domain walls investigated. The horizontal lines show the 10-90\%-levels of the transition from a transverse to a vortex domain wall. (b) The inset shows an off-axis electron holography image of a transverse wall in a $27 \mathrm{~nm}$ thick Co 3/4-ring. The color code (online color) indicates the direction of the in-plane magnetization and the black lines represent directly the stray field. The stray field strength was measured at several distances inside the marked area. The data points show the stray field normalized to the saturation magnetization as a function of the distance $r$ from the ring edge for the wall shown in the inset. The line is a $1 / r$-fit

one wall type to the other involves overcoming a local energy barrier [32], since the nucleation of the vortex core is associated with a strong twisting of the spins in the core region [43]. In arrays of interacting rings, the edge-to-edge spacing dependent stray field stabilizes transverse walls so that for small spacings (corresponding to a strong stray field from the adjacent domain wall) transverse walls are favored (Fig. 5(b)). For increasing spacing, the influence of the stray field from an adjacent wall is reduced, until vortex walls are formed in the rings with the lowest energy barrier for the vortex core nucleation. The further the spacing increases the more rings nucleate vortex walls (Fig. 5(c)). Thus the spacing at which a wall switches from transverse to vortex is related to the nucleation barrier, which depends on local imperfections such as the edge roughness. So the number of domain walls that have switched from transverse to vortex as a function of the edgeto-edge spacing is a measure of the distribution of energy barriers for the vortex core nucleation.

For NiFe, a relatively sharp transition occurs from all walls being transverse to all walls being vortex walls. This corresponds to a narrow energy barrier distribution, while the domain walls in Co rings exhibit a much wider transition. This difference is thought to result from the different polycrystalline microstructures of the $\mathrm{NiFe}$ (magnetically soft fcc crystallites with 
negligible anisotropy) and the Co (hcp crystallites with strong uniaxial anisotropy leading to a larger number of pinning sites). Furthermore, this results in the presence of transverse walls in our Co sample even at infinite spacings. Thus, we chose Co for the electron holography measurements rather than $\mathrm{NiFe}$ in order to be able to observe a transverse wall and its stray field in an isolated structure.

\subsection{Direct Observation of the Domain Wall Stray Field}

This spacing-dependent distribution for the vortex core nucleation needs to be transformed to a distribution as a function of the stray field strength, which is in a first approximation proportional to the energy. To do this, the stray field as well as the magnetization of the domain wall was imaged using off-axis electron holography. The inset of Fig. 6(b) shows an image of the inplane magnetic induction integrated in the electron beam direction, obtained from a transverse wall in a $27 \mathrm{~nm}$ thick isolated Co 3/4-ring designed with the same width as that of the rings imaged by XMCD-PEEM. No significant difference between the functional dependence of the stray ficld on the spacing is expected for a $27 \mathrm{~nm}$ and a $30 \mathrm{~nm}$ thick sample. The stray field was measured along the length of the region indicated in the image, and is shown as a function of the distance $r$ from the ring edge in Fig. 6(b), normalized to the saturation magnetization of Co. The line is a $1 / r$-fit which can be expected for the distance dependence of the stray field created by an area of magnetic poles for small $r$ [44]. This dependence also confirms earlier results from indirect Kerr effect measurements [23]. In order to obtain the stray field of one single domain wall acting on an adjacent wall, the stray field of an isolated wall was imaged.

The spacing-dependent energy barrier distribution is now rescaled to a field-dependent distribution using the measured stray field decay of Fig. 6(b) and presented in Fig. 7(a). The rescaled data can be fitted with the error function $\operatorname{erf}(x)$, which is the integral of a Gaussian distribution. The error function is not the only possible fit covered by the error bars in Fig. 7(a), but it is consistent with our data. Assuming a similar dependence of the stray field for $\mathrm{NiFe}$ like measured for $\mathrm{Co}$, a Gaussian distribution is also obtained for the energy barrier height distribution for $\mathrm{NiFe}$ as presented in Fig. 7(b). Thus a Gaussian distribution for the energy barriers is found, which is in agreement with the presence of independent local pinning centers at the particular wall position that determine the nucleation barrier. The position of the maximum is $H_{\max } / M_{\mathrm{s}}=0.21 \pm 0.10$ and the full width at half maximum $w / M_{\mathrm{s}}=0.16 \pm 0.05$, where $M_{\mathrm{s}}$ is the saturation magnetization. Using $E_{\max }=$ $\frac{1}{2} \mu_{0} M_{\mathrm{s}} H_{\max }$, an energy density of $E_{\max }=(8.4 \pm 4.0) \times 10^{4} \mathrm{~J} / \mathrm{m}^{3}$ equivalent to the field $H_{\max }$ can be obtained for the $30 \mathrm{~nm}$ thick Co sample.

Figure 6(a) shows that the transition for the Co sample saturates at a finite value for large spacings. In terms of the model described above, which explains how vortex walls are formed during relaxation from saturation, this 

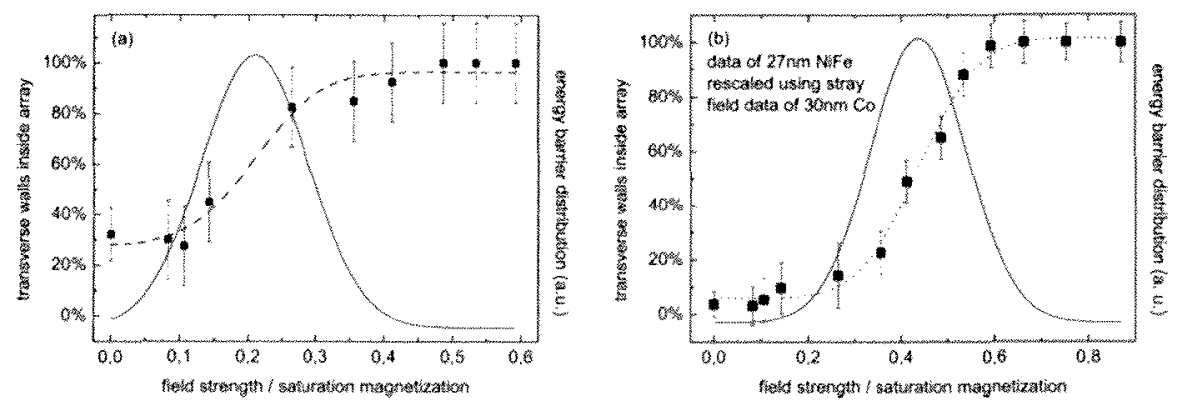

Fig. 7. (Color online, partly from [42]) (a) Black squares represent the same data points as shown in Fig. 6(a) for the $30 \mathrm{~nm}$ thick Co sample, but as a function of the normalized field strength. The black dashed line shows a fit with the error function. The corresponding Gaussian distribution of the energy barriers is shown as a full red line (online color). (b) Corresponding data for the $27 \mathrm{~nm}$ thick NiFe sample rescaled using the stray field measurement of a domain wall in $30 \mathrm{~nm}$ thick Co

means that an additional effective field would be needed to overcome the pinning of the remaining transverse walls at structural imperfections and to allow the vortex core nucleation and formation of an energetically favorable vortex wall. Since the pinning is much stronger in our Co sample than in the $\mathrm{NiFe}$ sample, this occurs here only for Co.

\section{Conclusion}

In conclusion, we have determined the spin structure of domain walls in $\mathrm{NiFe}$ and $\mathrm{Co}$ and extracted the corresponding room temperature phase diagrams with two phase boundaries between vortex walls for thick and wide as well as ultrathin rings and transverse walls for thin and narrow rings.

Using temperature dependent XMCD-PEEM imaging, we have observed a thermally activated switching from transverse walls established at room temperature to vortex walls at a transition temperature between $260^{\circ} \mathrm{C}$ and $310^{\circ} \mathrm{C}$ for $\mathrm{NiFe}$ rings. This gives direct experimental evidence for the fact that transverse and vortex walls are separated by an energy barrier, which can be overcome thermally. The low thickness regime of the phase diagrams revealed a second phase boundary which we attribute to spatial modulations of the magnetic properties in our thinnest samples.

Furthermore, we have mapped the stray field of a domain wall directly using off-axis electron holography with sub- $10 \mathrm{~nm}$ resolution, and we find that the field strength falls off with a $1 / r$-dependence. For interacting domain walls in $350 \mathrm{~nm}$ wide ring structures we observe a transition from a transverse to a vortex spin structure with increasing edge-to-edge spacing. By correlating this transition with the measured stray field, we are able to obtain the energy 
barrier height distribution for vortex core nucleation in our Co samples. This distribution has a Gaussian profile with the mean value being equivalent to an energy density of $(8.4 \pm 4.0) \times 10^{4} \mathrm{~J} / \mathrm{m}^{3}$.

\section{Acknowledgements}

The authors acknowledge support by the Deutsche Forschungsgemeinschaft through SFB 513, the Landesstiftung Baden-Württemberg, the EU through the European Regional Development Fund (Interreg III A Program), the EPSRC (U.K.), the Royal Society (U.K.), and by the EC through the 6th Framework Program. Part of this work was carried out at the Swiss Light Source, Villigen (Switzerland) and at Elettra, Trieste (Italy).

\section{References}

[1] D. A. Allwood et al., Science 309, 1688 (2005)

[2] G. A. Prinz, J. Magn. Magn. Mater. 200, 57 (1999)

[3] S. S. P. Parkin: U.S. patent 6,834,005 and patent application 10/984,055 (2004)

[4] P. M. Levy and S. Zhang, Phys. Rev. Lett. 79, 5110 (1997)

[5] U. Ebels et al., Phys. Rev. Lett. 84, 983 (2000)

[6] M. Kläui et al., Phys. Rev. Lett. 90, 097202 (2003)

[7] D. Atkinson et al., Nature Mat. 2, 85 (2003)

[8] Y. Nakatani, A. Thiaville, and J. Miltat, Nature Mat. 2, 521 (2003)

[9] R. Wieser, U. Nowak, and K. D. Usadel, Phys. Rev. B 69, 064401 (2004)

[10] M. Kläui et al., Appl. Phys. Lett. 87, 102509 (2005)

[11] U. Rüdiger et al., Phys. Rev. Lett. 80, 5639 (1998)

[12] A. D. Kent et al., J. Phys.: Cond. Mat. 13, R461 (2001)

[13] A. Yamaguchi et al., Phys. Rev. Lett. 92, 077205 (2004)

[14] M. Kläui et al., Phys. Rev. Lett. 94, 106601 (2005)

[15] M. Kläui et al., Phys. Rev. Lett. 95, 026601 (2005)

[16] D. Atkinson and R. P. Cowburn, Appl. Phys. Lett. 85, 1386 (2004)

[17] D. Lacour et al., Appl. Phys. Lett. 85, 4681 (2004)

[18] A. Yamaguchi et al., Appl. Phys. Lett. 86, 012511 (2005)

[19] N. Kazantseva, R. Wieser, and U. Nowak, Phys. Rev. Lett. 94, 037206 (2005)

[20] J. Rothman et al., Phys. Rev. Lett. 86, 1098 (2001)

[21] R. D. McMichael and M. J. Donahue, IEEE Trans. Magn. 33, 4167 (1997)

[22] X. Zhu et al., J. Appl. Phys, 93, 8540 (2003)

[23] M. Kläui et al., Appl. Phys. Lett. 86, 032504 (2005)

[24] L. J. Heyderman et al., J. Appl. Phys. 93, 10011 (2003)

[25] Y. G. Yoo et al., Appl. Phys. Lett. 82, 2470 (2003)

[26] J. Stöhr et al., Science 259, 658 (1993)

[27] L. J. Heyderman et al., J. Magn. Magn. Mater. 290-291, 86 (2005)

[28] A. Tonomura, Adv. Phys. 41, 59 (1992)

[29] R. E. Dunin-Borkowski et al., J. Microsc. 200, 187 (2000)

[30] M. Laufenberg et al., Appl. Phys. Lett. 88, 052507 (2006)

[31] M. Kläui et al., Appl. Phys. Lett. 85, 5637 (2004) 
[32] M. Kläui et al., Physica B 343, 343 (2004)

[33] The OOMMF package is available at $h$ ttp://math.nist.gov/oommf.

[34] C. A. F. Vaz et al., Phys. Rev. B 72, 224426 (2005)

[35] C. A. F. Vaz et al., Nucl. Instrum. Meth. Phys. Res. B 246, 13 (2006)

[36] M. Kläui et al., J. Appl. Phys. (in press) 99 (2006)

[37] I. Hashim, H. S. Joo, and H. A. Atwater, Surf. Rev. Lett. 2, 427 (1994)

[38] A. Hubert and R. Schäfer: Magnetic Domains - The Analysis of Magnetic Microstructures (Springer, Berlin Heidelberg New York 1998)

[39] M. H. Park et al., Phys. Rev. B 73, 094424 (2006)

[40] M. Kläui, et al., submitted

[41] Y. Nakatani et al, J. Magn. Magn. Mater. 290-291, 750 (2005)

[42] M. Laufenberg et al., submitted

[43] R. P. Cowburn et al., Phys. Rev. Lett. 83, 1042 (1999)

[44] M. McCaig: Permanent Magnets in Theory and Practice, 1st ed. (Pentech, London 1977) 